\title{
Biopharmaceutics Classification System: A Regulatory Approach
}

\author{
B. Basanta Kumar Reddy ${ }^{1, *}$ and A. Karunakar ${ }^{2}$ \\ ${ }^{\prime}$ Regulatory Affair Dpt., Indchemie Health Specialities Pvt. Ltd., (Alkem Research Laboratory), \\ M.I.D.C, Taloja, Dist-Raigarh, Navi Mumbai Maharashtra-410208, India \\ ${ }^{2}$ Regulatory Affair Department, IPDO, Dr. Reddy's Laboratory, Bachupally, Hyderabad, Andhra \\ Pradesh-502325, India
}

e-mail:bbasanthdreddy@gmail.com

\begin{abstract}
The Biopharmaceutics Classification System (BCS) is the result of continuous efforts in mathematical analysis for the elucidation of the kinetics and dynamics of the drug process in the gastrointestinal tract (GIT) for NDA (New Drug Application) and ANDA (Abbreviated New Drug Application) filings and biowaivers. This step reduces timelines in the new drug development process, both directly and indirectly, reduces unnecessary drug exposure in healthy volunteers, and increases impact for the replacement of certain bioequivalence (BE) studies with in vitro dissolution tests.
\end{abstract}

\section{INTRODUCTION}

A n ANDA (21 CFR 314) contains data for the review and ultimate approval of a generic drug product. Generic drug applications are termed "abbreviated" because they generally are not required to include preclinical (animal) and clinical (human) data to establish safety and effectiveness. Instead, generic applicants must scientifically demonstrate that their products are bioequivalent (i.e., performs in the same manner as the innovator drug).

Bioequivalence studies are conducted on generic drug products in place of animal studies, clinical studies, or bioavailability studies. In vitro-in vivo correlation (IVIVC) studies can be used in the development of new pharmaceuticals to reduce the number of human studies during formulation development. The main objective of an IVIVC is to serve as a surrogate for in vivo bioavailability and to support biowaivers. IVIVCs could also be employed to establish dissolution specifications and to support and validate the use of dissolution methods. This is because the IVIVC includes in vivo relevance to in vitro dissolution specifications.

The introduction of the Biopharmaceutics Classification System (BCS) in 1995 was the result of continuous efforts on mathematical analysis for the elucidation of the kinetics and dynamics of the drug process in the gastrointestinal (GI) tract (1). Since the BCS was introduced, it has been used as a regulatory tool for the replacement of certain BE studies with accurate in vitro dissolution tests. This step certainly reduces timelines in the drug development process, both directly and indirectly, and reduces unnecessary drug exposure in healthy volunteers, which is the normal study population in BE studies.

${ }^{*}$ Corresponding author.

\section{OBJECTIVES AND CONCEPT OF BCS}

The objectives of the BCS are (2):

- To improve the efficiency of the drug development and review process by recommending a strategy for identifying expendable clinical bioequivalence test.

- To recommend a class of immediate-release (IR) solid oral dosage forms for which bioequivalence may be assessed based on in vitro dissolution tests.

- To recommend methods for classification according to dosage form dissolution along with the solubilitypermeability characteristics of the drug product.

The BCS, which is based on scientific principles, presents a new paradigm in bioequivalence. According to the tenets of the BCS, certain drug products can be considered for biowaivers (i.e., product approval based on in vitro dissolution tests rather than bioequivalence studies in human subjects). At first, biowaivers were only applied to scale-up and postapproval changes (SUPAC) (3), but later the biowaiver principle was extended to the approval of new generic drug products. As a result, unnecessary human experiments can be avoided, and the cost of developing generic products can be significantly lowered (4). It provides drug designers an opportunity to manipulate the structure or physicochemical properties of lead candidates to achieve better "deliverability" (5).

\section{CLASSIFICATIONS}

The BCS is a scientific framework for classifying a drug substance based on its aqueous solubility and intestinal permeability (6). It allows for the prediction of in vivo pharmacokinetics of oral immediate-release (IR) drug products by classifying drug compounds into four classes (Table 1) based on their solubility related to dose and intestinal permeability in combination with the dissolution properties of the dosage form $(7,8)$.

The interest in this classification system stems largely from its application in early drug development and then in 
Table 1. The Biopharmaceutics Classification System

\begin{tabular}{lcc}
\hline Class & Solubility & Permeability \\
\hline I & High & High \\
\hline II & Low & High \\
\hline III & High & Low \\
\hline IV & Low & Low \\
\hline
\end{tabular}

the management of product change through its life cycle. In early drug development, knowledge of the class of a particular drug is an important factor influencing the decision to continue or stop its development. Therefore, an organization wishing to produce oral dosage forms will wish to limit development to molecules with high permeability. Increasingly, these considerations are incorporated from the very earliest phases, with the concept of property-based design being used in combinatorial chemistry to target production of compounds showing optimal properties.

This classification is associated with a drug dissolution and absorption model, which identifies the key parameters controlling drug absorption as a set of dimensionless numbers $(5,7)$ :

Absorption Number $\left(A_{n}\right)$ : Defined as the ratio of the mean residence time to mea $\mathrm{n}$ absorption time. It denotes the dimensionless dose/solubility ratio for the particular drug formulation. The dose/solubility ratio indicates whether the capacity of the Gl fluid is sufficient to dissolve the entire dose administered.

$$
A_{\mathrm{n}}=P_{\text {eff }} \times t_{\text {res }} / R
$$

Dissolution Number $\left(D_{n}\right)$ : Defined as the ratio of mean residence time to mean dissolution time.

$$
D_{\mathrm{n}}=t_{\text {res }} / t_{\text {Diss }}
$$

Dose Number $\left(D_{0}\right)$ : Defined as the mass divided by the product of uptake volume $(250 \mathrm{~mL})$ and solubility of drug.

$$
\mathrm{D}_{0}=M_{0} / C_{\mathrm{s}} V_{0}
$$

where $M_{0}$ is the dose of drug administered, $V_{0}$ is the initial gastric volume $(\approx 250 \mathrm{~mL}), C_{s}$ is the saturation solubility, $t_{\text {res }}$ is the mean residence time ( $\approx 180 \mathrm{~min}), t_{\text {diss }}$ is the time required for a drug particle to dissolve, $P_{\text {eff }}$ is the effective permeability, and $R$ is the radius of the intestinal segment.

\section{Class I}

The drugs of this class exhibit high absorption number and high dissolution number. The rate-limiting step is drug dissolution, and if dissolution is very rapid, then the gastric-emptying rate becomes the rate-determining step. These compounds are well absorbed, and their absorption rate is usually higher than the excretion rate $(7,9)$. Examples include metoprolol, diltiazem, verapamil, and propranolol.

\section{Class II}

The drugs of this class have a high absorption number but a low dissolution number. In vivo drug dissolution is then a rate-limiting step for absorption except at a very high dose number. The absorption for Class II drugs is usually slower than for Class I and occurs over a longer period of time. In vitro-in vivo correlation (IVIVC) is usually accepted for Class I and Class II drugs. The bioavailability of these products is limited by their solvation rates. Hence, a correlation between the in vivo bioavailability and the in vitro solvation can be found $(7,9,10)$. Examples include glibenclamide, phenytoin, danazol, mefenamic acid, nifedinpine, ketoprofen, naproxen, carbamezapine, and ketoconazole.

\section{Class III}

Drug permeability is the rate-limiting step for drug absorption, but the drug is solvated very quickly. These drugs exhibit a high variation in the rate and extent of drug absorption. Since the dissolution is rapid, the variation is attributable to alteration of physiology and membrane permeability rather than the dosage form factors. If the formulation does not change the permeability or gastrointestinal duration time, then Class I criteria can be applied $(7,9,10)$. Examples include cimetidine, ranitidine, acyclovir, neomycin $\mathrm{B}$, atenolol, and captopril.

\section{Class IV}

The drugs of this class are problematic for effective oral administration. These compounds have poor bioavailability. They are usually not well absorbed through the intestinal mucosa, and a high variability is expected. Fortunately, extreme examples of Class IV compounds are the exception rather than the rule, and these are rarely developed and marketed. Nevertheless, several Class IV drugs do exist $(7,9,10)$. Examples include hydrochlorothiazide, taxol, and furosemide.

\section{BCS CLASS BOUNDARIES}

Class boundary parameters (i.e., solubility, permeability, and dissolution) are for easy identification and determination of BCS class $(2,4,11)$.

Solubility: A drug substance is considered highly soluble when the highest dose strength is soluble in $250 \mathrm{~mL}$ or less of water over a $\mathrm{pH}$ range of $1-7.5$ at $37^{\circ} \mathrm{C}(4,11,12,13)$.

Permeability: A drug substance is considered highly permeable when the extent of absorption in humans is greater than $90 \%$ of an administered dose, based on mass-balance or compared with an intravenous reference dose $(12,13)$.

Dissolution: A drug product is considered rapidly dissolving when $85 \%$ or more of the labeled amount of drug substance dissolves within 30 min using USP Apparatus 1 or 2 in a volume of $900 \mathrm{~mL}$ or less of buffer solutions $(12,13)$. 


\section{Determination of Solubility}

Solubility is the amount of a substance that has passed into solution when equilibrium is attained between the solution and excess (i.e., undissolved) substance at a given temperature and pressure.

Solubilities are determined by exposing an excess of solid (drug) to the liquid in question (water/buffer) and assaying after equilibrium has been established. It usually takes 60-72 $\mathrm{h}$ to establish equilibrium; however, sampling at earlier points is necessary (14). Solubilities cannot be determined by the precipitation method because of the so-called metastable (solubility) zone. The $\mathrm{pH}$-solubility profile of the drug is determined at $37 \pm 1{ }^{\circ} \mathrm{C}$ in aqueous medium in the $\mathrm{pH}$ range of $1-7.5$ (per FDA guidelines) or 1.2-6.8 (per WHO guidelines). A sufficient number of samples should be evaluated to accurately define the $\mathrm{pH}$-solubility profile. A minimum of three replicate solubility determinations in each $\mathrm{pH}$ condition should be carried out. Depending on study variability, additional replicates may be necessary to provide a reliable estimate of solubility. Standard buffer solutions described in the USP are considered appropriate for use in solubility studies. If these buffers are not suitable for physical or chemical reasons, other buffer solutions may be used. Solution $\mathrm{pH}$ should be verified after addition of the drug substance to a buffer. Methods other than the traditional shake-flask method, such as acid or base titration methods, can also be used with justification to support the ability of such methods to predict equilibrium solubility of the test drug substance.

A drug substance is considered highly soluble when the highest dose strength is soluble in $250 \mathrm{~mL}$ or less of aqueous medium over the $\mathrm{pH}$ range of $1-7.5$. The volume estimate of $250 \mathrm{~mL}$ is derived from the typical volume of water consumed during the oral administration of a dosage form, which is about 8 ounces. This boundary value is a reflection of the minimum fluid volume anticipated in the stomach at the time of drug administration. A sufficient number of $\mathrm{pH}$ conditions should be evaluated to accurately define the $\mathrm{pH}$-solubility profile. The number of $\mathrm{pH}$ conditions for a solubility determination depends upon the ionization characteristics of the test drug substance. A minimum of three replicate determinations of solubility at each pH condition should be carried out (7). Standard buffer solutions described in pharmacopeias are considered appropriate for use in solubility studies. If these are not suitable for physical or chemical reasons, other buffer solutions can also be used provided the solution $\mathrm{pH}$ is verified. The concentration of drug substance in selected buffers or $\mathrm{pH}$ conditions should be determined using a validated stability-indicating assay that can determine the drug substance in the presence of its degradation products. If degradation of drug is observed as a function of buffer composition or $\mathrm{pH}$, it should be taken into consideration.

Solubility can be measured as either a kinetic or a thermodynamic value. Kinetic solubility measurements

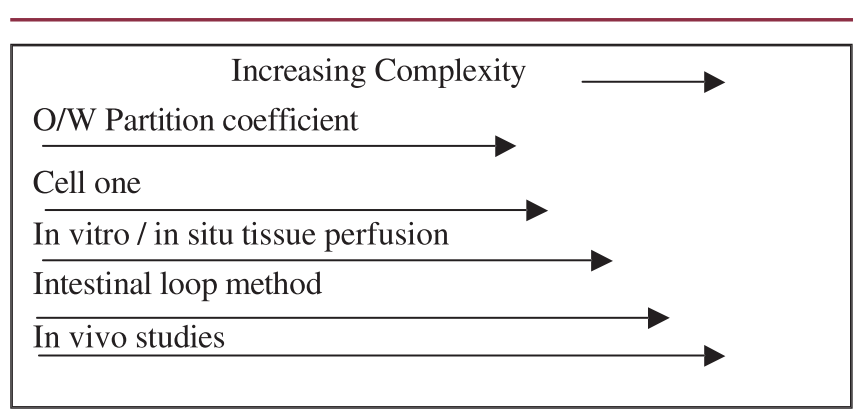

Figure 1. Complexities of permeability methods.

start from dissolved compound and represent the maximum (kinetic) solubility of the fastest precipitating species of a compound. Kinetic solubility values are strongly time-dependent. Due to the degree of supersaturation that may occur, values are likely to over-predict the thermodynamic solubility and are not expected to be reproducible between different kinetic methods, such as a turbidimetric-nephelometric method and UV absorption (15). In thermodynamics, solubility can predict drug properties during lead optimization. These methods include a scaled-down shake-flask method and a solvent evaporation method.

\section{Determination of Permeability}

The permeability is based directly on the extent of intestinal absorption of a drug substance in humans or indirectly on the measurements of the rate of mass transfer across the human intestinal membrane (3).

To understand the nature of gastrointestinal permeability limitations, there are methods and techniques to both screen and grade these characteristics. Figure 1 summarizes these techniques with their complexities (7). These methods range from a simple oil/water (O/W) partition coefficient to absolute bioavailability studies. A drug substance is considered highly permeable when the extent of absorption in humans is $90 \%$ or more of an administered dose, based on mass-balance or compared with an intravenous reference dose.

The methods that are routinely used for the determination of permeability include $(2,7)$ :

- Human studies

Mass balance pharmacokinetic studies

Absolute bioavailability studies, intestinal perfusion methods

- Intestinal permeability methods In vivo intestinal perfusions studies in humans In vivo or in situ intestinal perfusion studies in animals In vitro permeation experiments with excised human or animal intestinal tissue

- In vitro permeation experiments across epithelial cell monolayers (e.g., Caco-2 cells or TC-7 cells)

In mass-balance studies, unlabelled, stable isotopes or radiolabeled drug substances are used to determine the extent of drug absorption. However, this method 
gives highly variable estimates, and hence other methods are carried out. In absolute bioavailability studies, oral bioavailability is determined and compared with the intravenous bioavailability as a reference.

Intestinal perfusion models and in vitro methods are recommended for passively transported drugs. The observed low permeability of some drug substances in humans could be attributed to the efflux of drug by various membrane transporters like P-glycoprotein. This leads to misinterpretation of drug substance permeability. An interesting alternative to intestinal tissue models is the use of well-established in vitro systems based on the human adenocarcinoma cell line Caco-2. These cells serve as a model of small intestinal tissue. The differentiated cells exhibit the microvilli typical of the small intestinal mucosa and the integral membrane proteins of the brush-border enzyme. In addition, they form the fluidfilled domes typical of a permeable epithelium. Recent investigations of Caco- 2 cell lines have indicated their ability to transport ions, sugars, and peptides. The directed transport of bile acids and vitamin B- 12 across Caco- 2 cell lines has also been observed. These properties have established the Caco- 2 cell line as a reliable in vitro model of the small intestine (7).

\section{Determination of Dissolution}

Formulation composition and the manufacturing process generally influence in vitro drug dissolution. The BCS classifies a drug product as rapidly dissolving when no less than $85 \%$ of the labeled amount of the drug substance dissolves in 30 min using the following $(3,11)$ :

- USP Apparatus 1 (basket) at 100 rpm or USP Apparatus 2 (paddle) at $50 \mathrm{rpm}$.

- Dissolution medium volume of $900 \mathrm{~mL}$ or less in each of the following (13):

1. $0.1 \mathrm{~N} \mathrm{HCl}$ or simulated gastric fluid (SGF) USP without enzymes

2. A pH 4.5 buffer

3. A pH 6.8 buffer or simulated intestinal fluid (SIF) USP without enzymes $(3,11)$.

- The similarity factor $\left(f_{2}\right)$ for test versus reference profile comparisons should be greater than 50 (i.e., $f_{2}$ value between 50 and 100 suggests the two dissolution profiles are similar).

$$
f_{2}=50 \cdot \log \left\{\left[1+\frac{1}{n} \sum_{\mathrm{t}=1}^{n}\left(R_{t}-T_{t}\right)^{2}\right]^{-0.5} \times 100\right\}
$$

where $R_{t}$ and $T_{t}$ are the cumulative percentage dissolved at time point $t$ for reference and test products, respectively, and $n$ is the number of pool points (3).

According to the BCS guidance, the test and reference dissolution profiles are considered similar if both products have at least $85 \%$ dissolution in 15 min or if comparison of profiles by the $f_{2}$ test results in an $f_{2}$ value of at least 50 . To allow for the use of mean data, the coefficient of variation
Table 2. Physicochemical and Physiological Parameters Important to Drug Dissolution in the Gastrointestinal Tract

\begin{tabular}{|c|c|c|}
\hline Factor & $\begin{array}{l}\text { Physicochemical } \\
\text { Properties }\end{array}$ & $\begin{array}{l}\text { Physiological } \\
\text { Properties }\end{array}$ \\
\hline $\begin{array}{l}\text { Surface area of } \\
\text { drug }\end{array}$ & Particle size, wettability & $\begin{array}{l}\text { Surfactants in gastric } \\
\text { juice and bile }\end{array}$ \\
\hline Diffusivity of drugs & Molecular size & $\begin{array}{l}\text { Viscosity of luminal } \\
\text { contents }\end{array}$ \\
\hline $\begin{array}{l}\text { Boundary layer } \\
\text { thickness }\end{array}$ & $\begin{array}{l}\text { Concentration of } \\
\text { the drug }\end{array}$ & $\begin{array}{l}\text { Motility patterns and } \\
\text { flow rate }\end{array}$ \\
\hline Solubility & $\begin{array}{l}\text { Hydrophilicity, crystal } \\
\text { structure, solubilization }\end{array}$ & $\begin{array}{l}\mathrm{pH} \text {, buffer capacity, bile } \\
\text { and food composition }\end{array}$ \\
\hline $\begin{array}{l}\text { Amount of drug } \\
\text { already dissolved }\end{array}$ & $\begin{array}{l}\text { Hydrophilic, lipophilic } \\
\text { nature of the drug }\end{array}$ & Permeability \\
\hline $\begin{array}{l}\text { Volume of solvent } \\
\text { available }\end{array}$ & $\begin{array}{l}\text { Depends upon type } \\
\text { of body fluid }\end{array}$ & $\begin{array}{l}\text { Secretion, coadminis- } \\
\text { tered fluids }\end{array}$ \\
\hline
\end{tabular}

should not be more than $20 \%$ at earlier time points (e.g., $10 \mathrm{~min}$ ) and should not be more than $10 \%$ at other times (11).

Dissolution performance is influenced by both the physicochemical properties of the substance and the prevailing physiological conditions in the Gl tract, which varies between the fasted- and fed-states as well as within and among subjects. The key in vivo parameters influencing drug product dissolution performance are summarized in Table $2(5,16,17)$.

There was consensus that the $f_{2}$ test is not necessary when the two products each provide at least $85 \%$ dissolution in $30 \mathrm{~min}$. A profile comparison test (e.g., $f_{2}$ or a single time point comparison) would be necessary when at least one product has $85 \%$ dissolution between 30 and $60 \mathrm{~min}$. The number of time points sampled need not be extraordinary; sampling can be as infrequent as every $30 \mathrm{~min}$ (i.e., two samples over $60 \mathrm{~min}$ ). The $f_{2}$ acceptance criterion $\left(f_{2} \geq 50\right)$ can be lowered with justification that considers underlying biopharmaceutic characteristics and riskbased factors (e.g., dissolution results from the most relevant $\mathrm{pH})(11)$.

\section{REGULATORY APPLICATIONS INDs and NDAs}

BCS-based biowaivers are applicable to the to-bemarketed formulation when changes in components, composition, or method of manufacture occur to the clinical trial formulation, as long as the dosage forms have rapid and similar in vitro dissolution profiles. This approach is useful only when the drug substance is highly soluble and highly permeable (BCS Class I) and the pre- and post-change formulations are pharmaceutical equivalents. These are intended only for BE studies and are not applicable to food-effect BA studies or other pharmacokinetic studies (14). 


\section{ANDAs}

Biowaivers can be requested for rapidly dissolving immediate-release (IR) test products containing highly soluble and highly permeable drug substances if the reference listed drug (RLD) is also rapidly dissolving and the test products exhibit dissolution profiles similar to the RLD. This approach is useful when the test and reference dosage forms are pharmaceutical equivalents.

\section{Postapproval Changes}

Biowaivers can be requested for significant postapproval changes (e.g., Level 3 changes in components and compositions) to a rapidly dissolving, immediate-release (IR) product containing a highly soluble, highly permeable drug substance, provided that dissolution remains rapid for the post-change product and both pre- and postchange products exhibit similar dissolution profiles. The BCS enables pharma manufacturers to reduce the cost of scale-up and postapproval changes to certain oral drug products (rapidly dissolving drug products of Class I drug).

\section{Request for Biowaivers}

The BCS-based biowaivers apply during both pre- (IND/ NDA and ANDA) and postapproval phases. Considering the uncertainties associated with in vitro dissolution tests, the proposed biowaivers are as follows $(8,12)$.

\section{Data Supporting High Solubility}

Data supporting high solubility of the test drug substance should include:

- A description of test methods including information on analytical methods and composition of the buffer solutions.

- Chemical structure, molecular weight, nature of the drug substance (acid, base, amphoteric, or neutral), and dissociation constants.

- Test results (mean, standard deviation, and coefficient of variation) summarized in a table under solution $\mathrm{pH}$, drug solubility (e.g., $\mathrm{mg} / \mathrm{mL}$ ), and volume of media required to dissolve the highest dose strength.

- A graphic representation of mean $\mathrm{pH}$-solubility profile.

\section{Data Supporting High Permeability}

Data supporting high permeability of the test drug substance should include:

- For human pharmacokinetic studies, information on study design and methods used along with the pharmacokinetic data.

- For direct permeability methods, information supporting the suitability of a selected method that encompasses a description of the study method; criteria for selection of human subjects, animals, or epithelial cell line; drug concentrations in the donor fluid; description of the analytical method and method used to calculate extent of absorption or permeability; and where appropriate, information on efflux potential (e.g., bidirectional transport data).
- A list of selected model drugs along with data on extent of absorption in humans (mean, standard deviation, coefficient of variation) used to establish suitability of a method, permeability values for each model drug (mean, standard deviation, coefficient of variation), permeability class of each model drug, and a plot of the extent of absorption as a function of permeability (mean \pm standard deviation or $95 \%$ confidence interval) with identification of the low/high permeability class boundary and selected internal standard. Information to support high permeability of a test drug substance should include permeability data on the test drug substance, the internal standards (mean, standard deviation, and coefficient of variation), stability information, data supporting passive transport mechanism where appropriate, and methods used to establish high permeability of the test drug substance.

\section{Data Supporting Rapid and Similar Dissolution}

Data supporting rapid dissolution attributes of the test and reference products should include:

- A brief description of the products used for dissolution testing, including information on batch or lot number, expiry date, dimensions, strength, and weight.

- Dissolution data obtained with 12 individual units of the test and reference products using recommended test methods. The percentage of label claim dissolved at each specified testing interval should be reported for each individual dosage unit. The mean percent dissolved, range (highest and lowest) of dissolution, and coefficient of variation (relative standard deviation) should be tabulated. A graphic representation of the mean dissolution profiles for the test and reference products in the three media should also be included.

- Data supporting similarity in dissolution profiles between the test and reference products in each of the three media, using the $f_{2}$ metric.

The in vivo absorbability of drugs categorized as BCS Class II is very difficult to predict because of the large variability in the absorption or dissolution kinetics and the lack of an adequate in vitro system for evaluating the dissolution behavior. For example, to predict the in vivo absorption kinetics of griseofulvin (categorized as BCS Class II), it is orally administrated as a powder to rats, based on the Gastrointestinal-Transit-Absorption model (GITA model), which consists of the absorption, dissolution, and Gl-transit processes. Using the dissolution rate constants $\left(K_{\text {dis }}\right)$ of griseofulvin obtained with FaSSIF (fasted-state simulated intestinal fluid), FeSSIF (fed-state simulated intestinal fluid), and other simulated media, simulation lines did not describe the observed mean plasma profile at all.

Permeability of prodrugs will depend on the mechanism and (anatomical) site of conversion to the drug substance. When the prodrug-to-drug conversion occurs predominantly after intestinal membrane permeation, the 
permeability of the prodrug should be measured. When this conversion occurs before intestinal permeation, the permeability of the drug should be determined. Dissolution and $\mathrm{pH}$-solubility data on both prodrug and drug can be relevant. Sponsors may wish to consult with appropriate review staff before applying the BCS approach to IR products containing prodrugs.

For in vivo relative bioavailability studies, dissolution should be greater than $85 \%$ in 30 min in the three recommended dissolution media.

For in vivo bioequivalence, test and reference products should exhibit similar dissolution profiles under the dissolution test conditions defined for rapidly dissolving products.

When both the test and the reference products dissolve $85 \%$ or more of the label amount in less than $15 \mathrm{~min}$ in all three dissolution media, then a profile comparison is unnecessary.

Excipients used in the dosage form should have been previously used or currently FDA approved IR solid dosage forms. The quantity of excipients in the IR product should be consistent with their intended functions. When new excipients or atypically large amounts of commonly used excipients are included in an IR solid dosage form, additional information documenting the absence of an impact on BA of the drug may be requested by the agency. Such information can be provided with a relative BA study using a simple aqueous solution as the reference product. Large quantities of certain excipients, such as surfactants (e.g., polysorbate 80) and sweeteners (e.g., mannitol or sorbitol), may be problematic, and sponsors are encouraged to contact the review division when this is a factor.

The drug must be stable in gastrointestinal tract, and the product designed not to be absorbed in oral cavity.

All other application commitments should be met.

\section{CONCLUSION}

The Biopharmaceutics Classification System provides a regulatory tool for replacing certain bioequivalence studies with accurate in vitro dissolution tests during the process of generic drug development. Considering the uncertainties associated with in vitro dissolution tests, the BCS proposed biowaivers for rapidly dissolving drug products (i.e., a drug must be stable in the gastrointestinal tract), non-narrow therapeutic index drugs, and other application commitments should be met during drug development.

\section{REFERENCES}

1. Rinaki, E.; Valsami, G.; Macheras, P. Quantitative biopharmaceutics classification system:The central role of dose/solubility ratio. Pharm. Res. 2003, 20 (12), 1917-1925.

2. Waiver of In Vivo Bioavailability and Bioequivalence Studies for Immediate-Release Solid Oral Dosage Forms Based on a Biopharmaceutics Classification System;
Guidance for Industry; U.S. Department of Health and Human Services, Food and Drug Administration, Center for Drug Evaluation and Research (CDER), U.S. Government Printing Office:Washington, DC, August 2000. http://www.fda.gov/downloads/Drugs/ GuidanceComplianceRegulatorylnformation/ Guidances/ucm070246.pdf (accessed Jan 11, 2011).

3. Yu, L. X.; Amidon, G. L.; Polli, J. E.; Zhao, H.; Mehta, M. U.; Conner, D. P.; Shah, V. P.; Lesko, L. J.; Chen, M. L.; Lee, V. H. L. Biopharmaceutics classification system:The scientific basis for biowaiver extensions. Pharm. Res. 2002, 19 (7), 921-925.

4. Lindenberg, M.; Kopp, S.; Dressman, J. B. Classification of orally administered drugs on the World Health Organization model list of essential medicines according to the biopharmaceutics classification system. Eur. J. Pharm. Biopharm. 2004, 58, 265-278.

5. Varma, M. V.; Khandavilli, S.; Ashokraj, Y.; Dhanikula, A.; Sood, A.; Thomas, N. S.; Pillai, O.; Sharma, P.; Gandhi, R.; Agrawal, S.; Nair, V.; Panchagnula, R. Biopharmaceutic classification system: A scientific framework for pharmacokinetic optimization in drug research. Curr. Drug Metab. 2004, 5 (5), 375-388.

6. Amidon, G. L.; Lennernas, H.; Shah, V.P.; Crison, J. R. A. A theoretical basis for a biopharmaceutic drug classification:The correlation of in vitro drug product dissolution and in vivo bioavailability. Pharm. Res. 1995, 12 (3), 413-419.

7. Gothoskar, A.V.Biopharmaceutical classification of drugs Pharm. Rev. [Online] 2005, 3 (1). http://www.pharmainfo. net/reviews/biopharmaceutical-classification-drugs (accessed Jan 18, 2011).

8. Hussain, A. S.; Lesko, L. J.; Lo, K. Y.; Shah, V. P.; Volpe, D.; Williams, R. L. The Biopharmaceutics Classification System: Highlights of the FDA's Draft Guidance. Dissolution Technol. 1999, 6 (2), 5-9. http://www. dissolutiontech.com/DTresour/599articles/Biopharm Class2_copy.html (accessed Jan 18, 2011).

9. Devane, J. Oral drug delivery technology: Addressing the solubility/permeability paradigm. Pharm. Technol. 1998, 22, 68-74.

10. Lipka, E.; Amidon, G. L. Setting bioequivalence requirements for drug development based on preclinical data: Optimizing oral drug delivery systems. J. Controlled Release. 1999, 62, 41-49.

11. Polli, J.E.; Yu, L. X.; Cook, J. A.; Amidon, G. L.; Borchardt, R.T.; Burnside, B. A.; Burton, P. S.; Chen, M. L.; Conner, D. P.; Faustino, P. J.; Hawin, A.; Hussain, A. S.; Joshi, H. N.; Kwei, G.; Lee, V. H. L.; Lesko, L. J.; Lipper, R. A.; Loper, A. E.; Nerurkar, S. G.; Polli, J.W.; Sanvordeker, D. R.;Taneja, R.; Uppoor, R. S.; Vattikonda, C. S.; Wilding, I.; Zhang, G. Summary workshop report: Biopharmaceutics classification system-implementation challenges and extension opportunities. J. Pharm. Sci. 2004, 93 (6), 1375-1381.

12. Gohel, M.C. An audit of recent inputs on biopharmaceutical classification system. Pharm. Rev. [Online] 
2005, 3 (1). http://www.pharmainfo.net/reviews/ audit-recent-inputs-biopharmaceutical-classificationsystem (accessed Jan 18, 2011).

13. United States Pharmacopeia and National Formulary USP 23-NF 18; The United States Pharmacopeial Convention, Inc.: Rockville, MD, 1994.

14. Sacham, N. K.; Bhattacharya, A.; Pushkar, S.; Mishra, A. Biopharmaceutical classification system: A strategic tool for oral drug delivery technology. Asian J. Pharm. 2009, 3 (2), 76-81.
15. Stuart, M.; Box, K. Chasing equilibrium: measuring the intrinsic solubility of weak acids and bases. Anal. Chem. 2005, 77 (4), 983-990.

16. Blume, H. H.; Schug, B. S. The biopharmaceutics classification system (BCS): Class III drugs better candidates for BA/BE waiver? Eur. J. Pharm. Sci. 1999, $9(2), 117-121$.

17. Dressman, J. B.; Reppas, C. In vivo-in vitro correlations for lipophilic, poorly water-soluble drugs. Eur.J. Pharm. Sci. 2000, 11 (Suppl. 2), S73-S80. 